\title{
NECESSARY AND SUFFICIENT CONDITIONS FOR HOMOTOPY CLASSIFICATION BY COHOMOLOGY AND HOMOTOPY HOMOMORPHISMS
}

\author{
MARTIN STERNSTEIN ${ }^{1}$
}

\begin{abstract}
Using obstruction theory tools to a pair of spaces two invariants are defined whose vanishing is a necessary and sufficient condition for homotopy classification of certain maps between these spaces by the induced cohomology and homotopy homomorphisms respectively.
\end{abstract}

1. Introduction. By the Hopf Theorem the set of homotopy classes of maps from a complex of dimension $n$ into an $n$-sphere can be classified by the cohomology homomorphism each class induces. To a pair of spaces Emery Thomas [8] has defined invariants whose vanishing implies that a map between these spaces is null-homotopic if all of its induced cohomology homomorphisms are zero. To a pair of spaces Donald Kahn [1] has assigned groups whose vanishing implies that a map between these spaces is null-homotopic if all of its induced homotopy homomorphisms are zero. For these classification problems we will give necessary and sufficient conditions from which the above sufficiency results will follow.

2. Definitions. All spaces in this paper are pathwise connected, basepointed, and have the homotopy type of CW-complexes. All maps and homotopies will preserve basepoints. $Y$ will be simply connected, and we use the following notation for maps arising from a fixed homotopy decomposition $\left\{Y_{n}\right\}$ of $Y$ :

$$
p_{n}: Y_{n} \rightarrow Y_{n-1}, \quad \beta_{n}: Y \rightarrow Y_{n}, \quad q_{j n}: Y_{n} \rightarrow Y_{j},
$$

Presented to the Society, January 17, 1972; received by the editors July 13, 1971. AMS 1970 subject classifications. Primary 55G37, 55G35, 55G45.

Key words and phrases. Posnikov decomposition, $k$-invariant, higher order cohomology operation, obstruction.

${ }_{1}$ The preparation of this paper was sponsored in part by a NSF graduate fellowship. This paper is a condensation of the author's Ph.D. dissertation at Cornell University.

(c) American Mathematical Society 1972 


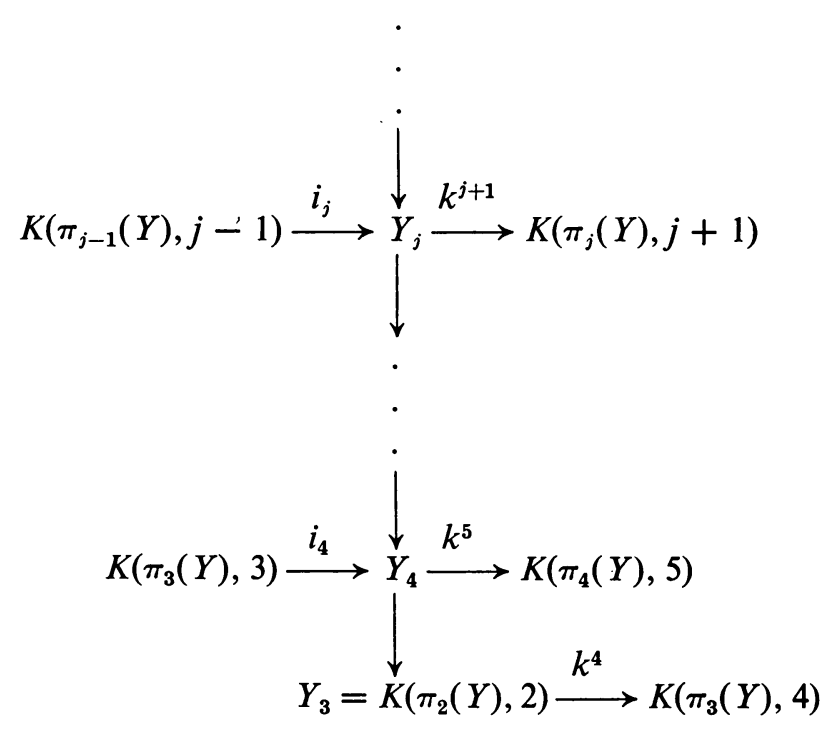

where the $\left\{k^{j}\right\}$ are the $k$-invariants and $i_{j}$ is the inclusion of the fiber into the total space of the fibration $p_{j}: Y_{j} \rightarrow Y_{j-1}$. We also have

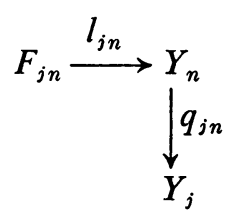

where $l_{j n}$ is the inclusion of the fiber into the total space of the fibration $q_{j n}: Y_{n} \rightarrow Y_{j}$.

$k_{j}^{n+1}=l_{j n}^{*} k^{n+1} \in H^{n+1}\left(F_{j n} ; \pi_{n}(Y)\right)$ defines a higher order cohomology operation as follows. Let $v^{j} \in H^{j}\left(X ; \pi_{j}\left(F_{j n}\right)\right)$ and we set

$$
k_{j}^{n+1} \vdash v^{j}=\left\{h^{*}\left(k_{j}^{n+1}\right) \mid h: X \rightarrow F_{j n} \text { where } h^{*}\left(e^{j}\right)=v^{j}\right\}
$$

where $e^{j} \in H^{j}\left(F_{j n} ; \pi_{j}\left(F_{j n}\right)\right)$ is the fundamental class of $F_{j n}$. Note that $k_{n-1}^{n+1}$ is a primary cohomology operation.

Suppose $f_{j+1}, g_{j+1}: X \rightarrow Y_{j+1}$ with $p_{j+1} f_{j+1}=p_{j+1} g_{j+1}, g_{n}$ is any lifting of $g_{j+1}$ to $Y_{n}$, and either $Y$ is an $H$-space or $j>n-m+1$ where $j \geqq 2$, $m \geqq 2$ and $Y$ is $(m-1)$-connected. Then Olum's difference formula [4] gives that

$$
\begin{aligned}
\mathcal{O}^{n+1}\left(f_{j+1}\right)-\mathcal{O}^{n+1}\left(g_{n}\right) & =\mathcal{O}^{n+1}\left(f_{j+1}\right)-\mathcal{O}^{n+1}\left(g_{j+1}\right) \\
& =k_{j}^{n+1} \vdash\left(\mathcal{O}^{j}\left(f_{j+1}, g_{j+1}\right) \text { rel } p_{j+1}\right)
\end{aligned}
$$

where $\mathcal{O}^{n+1}\left(f_{j+1}\right)$ is the obstruction to lifting $f_{j+1}$ to $Y_{n+1}$ and $\mathcal{O}^{j}\left(f_{j+1}, g_{j+1}\right)$ 
is the $j$ th obstruction to a homotopy from $f_{j+1}$ to $g_{j+1}$. (A similar difference formula is given by Mizuno [2].)

Given a map $f: X \rightarrow Y$ we define $f_{(n)}=\beta_{n} f: X \rightarrow Y_{n}$.

If $\gamma^{n} \in H^{n}(Y ; G)$ we define $\gamma_{\sharp}^{n}: \pi_{n}(Y) \rightarrow G$ to be the composition of the Hurewicz homomorphism with the homomorphism on $H_{n}(Y)$ induced by $\gamma^{n}$. Now let $\gamma_{*}^{n}: H^{n}\left(X ; \pi_{n}(Y)\right) \rightarrow H^{n}(X ; G)$ be induced by $\gamma_{\sharp}^{n}$.

Olum [5] has shown that if $f, g: X \rightarrow Y$ with $\mathcal{O}^{n}(f, g)$ nonvoid, then for any $\gamma^{n} \in H^{n}(Y ; G)$,

$$
\left(f^{*}-g^{*}\right) \gamma^{n}=\gamma_{*}^{n} \mathcal{O}^{n}(f, g)
$$

And for any $u^{n} \in \mathcal{O}^{n}(f, g)$ and any $\gamma \in \pi_{n}(X)$,

$$
\left(f_{\sharp}-g_{\sharp}\right) \gamma=u_{\sharp}^{n} \gamma .
$$

Using the homomorphism $H^{j}(X ; G) \rightarrow \operatorname{Hom}\left(H_{j}(X), G\right)$ and the Hurewicz homomorphisms $\pi_{j}(X) \rightarrow H_{j}(X)$ and $\pi_{j}(Y) \rightarrow H_{j}(Y)$ we define

$$
\begin{aligned}
& \mathscr{H}_{j}: H^{j}\left(X ; \pi_{j}(Y)\right) \rightarrow H^{j}\left(X ; H_{j}(Y)\right), \\
& \mathscr{G}_{j}: H^{j}\left(X ; \pi_{j}(Y)\right) \rightarrow \operatorname{Hom}\left(\pi_{j}(X), \pi_{j}(Y)\right) .
\end{aligned}
$$

The map $i_{j}: K\left(\pi_{j-1}(Y), j-1\right) \rightarrow Y_{j}$ together with the isomorphism $H^{j-1}\left(X ; \pi_{j-1}(Y)\right) \rightarrow\left[X, K\left(\pi_{j-1}(Y), j-1\right)\right]$ defines a function

$$
\left(\left(i_{j}\right)\right): H^{j-1}\left(X ; \pi_{j-1}(Y)\right) \rightarrow\left[X, Y_{j}\right]
$$

$f^{*}=g^{*}$ will mean $f$ and $g$ induce the same cohomology homomorphisms over all finitely generated abelian coefficient groups.

3. Statements and proofs of main theorems. For Theorems 3.1-3.3 we have the hypothesis that $j \leqq n-1$ and either $Y$ is an $H$-space or $Y$ is $(m-1)$-connected and $j>n-m+1$.

THEOREM 3.1. Given $f: X \rightarrow Y_{n+1}$. Let $g: X \rightarrow Y_{n+1}$ be any map such that $f_{(j)} \cong g_{(j)}$. Then $f_{(j+1)}^{*}=g_{(j+1)}^{*} \Rightarrow f_{(j+1)} \cong g_{(j+1)}$ for all such $g$, if and only if

$$
\left[\operatorname{ker}\left(k_{j}^{n+1} \mid X\right) \cap \operatorname{ker} \mathscr{H}_{j}\right] / \mathcal{O}^{j}(f, f)=0 .
$$

Proof of 3.1. Suppose

$$
\left[\operatorname{ker}\left(k_{j}^{n+1} \mid X\right) \cap \operatorname{ker} \mathscr{H}_{j}\right] / \mathcal{O}^{j}(f, f)=0
$$

and we are given $g: X \rightarrow Y_{n+1}$ such that $g_{(j)} \cong f_{(j)}$ and $g_{(j+1)}^{*}=f_{(j+1)}^{*}$. $q_{j, n+1}: Y_{n+1} \rightarrow Y_{j}$ a fibration gives $\bar{g}: X \rightarrow Y_{n+1}$ with $g \cong \bar{g}$ and $\bar{g}_{(j)}=f_{(j)}$. Since $g \cong \bar{g}$ implies $g^{*}=\bar{g}^{*}$ we have $\bar{g}_{(j+1)}^{*}=f_{(j+1)}^{*} .0 \in \mathcal{O}^{n+1}\left(f_{(j+1)}\right)$ and $0 \in \mathcal{O}^{n+1}\left(\bar{g}_{(j+1)}\right)$ so the difference formula gives that

$$
\left\{\mathcal{O}^{j}\left(f_{(j+1)}, \bar{g}_{(j+1)}\right) \operatorname{rel} p_{j+1}\right\} \in \operatorname{ker}\left(k_{j}^{n+1} \mid X\right) .
$$

$H^{j}\left(Y ; H_{j}(Y)\right) \rightarrow \operatorname{Hom}\left(H_{j}(Y), H_{j}(Y)\right)$ is epi so there exists $h^{j} \in H^{j}\left(Y ; H_{j}(Y)\right)$ 
such that $h^{j}$ induces the identity $H_{j}(Y) \rightarrow H_{j}(Y)$ and thus $h_{*}^{j}=\mathscr{H}_{j}$. Using (2.1) we have

$$
0=\left(f_{(j+1)}^{*}-\bar{g}_{(j+1)}^{*}\right) h^{j}=h_{*}^{j} \mathcal{O}^{j}\left(f_{(j+1)}, \bar{g}_{(j+1)}\right)=\mathscr{H}_{j} \mathcal{O}^{j}\left(f_{(j+1)}, \bar{g}_{(j+1)}\right) .
$$

$\left\{\mathcal{O}^{j}\left(f_{(j+1)}, \bar{g}_{(j+1)}\right)\right.$ rel $\left.p_{j+1}\right\} \in \mathcal{O}^{j}\left(f_{(j+1)}, \bar{g}_{(j+1)}\right)$ which gives that

$$
\left\{\mathcal{O}^{j}\left(f_{(j+1)}, \bar{g}_{(j+1)}\right) \text { rel } p_{j+1}\right\} \in \operatorname{ker} \mathscr{H}_{j} \text {. }
$$

Using the hypothesis we have

$$
\left\{\mathcal{O}^{j}\left(f_{(j+1)}, \bar{g}_{(j+1)}\right) \text { rel } p_{j+1}\right\} \in \mathcal{O}^{j}(f, f)=\mathcal{O}^{j}\left(f_{(j+1)}, f_{(j+1)}\right) .
$$

$\left\{\mathcal{O}^{j}\left(f_{(j+1)}, \bar{g}_{(j+1)}\right)\right\}$, since nonempty, is a coset of $\left\{\mathcal{O}^{j}\left(f_{(j+1)}, f_{(j+1)}\right)\right\}$ and in fact is the same set since they have the common element

$$
\left\{\mathcal{O}^{j}\left(f_{(j+1)}, \bar{g}_{(j+1)}\right) \text { rel } p_{j+1}\right\} \text {. }
$$

Therefore since $0 \in\left\{\mathcal{O}^{j}\left(f_{(j+1)}, f_{(j+1)}\right)\right\}$ we have $0 \in\left\{\mathcal{O}^{j}\left(f_{(j+1)}, \bar{g}_{(j+1)}\right)\right\}$. So $f_{(j+1)} \cong \bar{g}_{(j+1)}$ and since $g \cong \bar{g}$ we have the desired result $f_{(j+1)} \cong g_{(j+1)}$.

Going in the other direction, let us suppose there exists a $v^{j} \in$ $H^{j}\left(X ; \pi_{j}(Y)\right)$ such that $v^{j} \in \operatorname{ker}\left(k_{j}^{n+1} \mid X\right) \cap \operatorname{ker} \mathscr{H}_{j}$ but $v^{j} \notin \mathcal{O}^{j}(f, f)$. There exists $\mu: K\left(\pi_{j}(Y), j\right) \times Y_{j+1} \rightarrow Y_{j+1}$ such that $\mu \mid K\left(\pi_{j}(Y), j\right) \vee Y_{j+1}=l_{j, j+1} \vee 1$ and $p_{j+1} \mu=p_{j+1} \rho_{2}$. $v^{j}$ defines a map $\bar{v}^{j}: X \rightarrow K\left(\pi_{j}(Y), j\right)$ such that $\left(\bar{v}^{j}\right)^{*} e^{j}=v^{j}$ where $e^{j}$ is the fundamental class of $K\left(\pi_{j}(Y), j\right)$. Let $\tilde{v}^{j}=$ $\mu\left(\bar{v}^{j}, f_{(j+1)}\right)$. By a standard calculation [3], we have

$$
\left\{\mathcal{O}^{j}\left(f_{(j+1)}, \tilde{v}^{j}\right) \text { rel } p_{j}\right\}=v^{j},
$$

so $f_{(j+1)} \cong \tilde{v}^{j}$ since by hypothesis $v^{j} \notin \mathcal{O}^{j}(f, f)$. However

$$
\left(f_{(j+1)}^{*}-\left(\tilde{v}^{j}\right)^{*}\right) \gamma^{j+1}=\gamma_{*}^{j+1} \mathcal{O}^{j}\left(f_{(j+1)}, \tilde{v}^{j}\right)=\gamma_{*}^{j+1}\left(v^{j}\right)=0
$$

for all $\gamma^{j+1}$ because $\mathscr{H}_{j}\left(v^{j}\right)=0$. Finally $v^{j} \in \operatorname{ker}\left(k_{j}^{n+1} \mid X\right)$ together with the difference formula gives that $0 \in \mathcal{O}^{n+1}\left(\tilde{v}^{j}\right)$ so $\tilde{v}^{j}$ lifts to $g: X \rightarrow Y_{n+1}$ with $f_{(j+1)}^{*}=g_{(j+1)}^{*}$ but $f_{(j+1)} \cong g_{(j+1)}$.

THeOREM 3.2. Given $f: X \rightarrow Y_{n+1}$. Let $g: X \rightarrow Y_{n+1}$ be any map such that $f_{(j)} \cong g_{(j)}$. Then $\left(f_{(j+1)}\right)_{\#}=\left(g_{(j+1)}\right)_{\#} \Rightarrow f_{(j+1)} \cong g_{(j+1)}$ for all such $g$, if and only if

$$
\left[\operatorname{ker}\left(k_{j}^{n+1} \mid X\right) \cap \operatorname{ker} \mathscr{G}_{j}\right] / \mathcal{O}^{j}(f, f)=0 .
$$

The proof of the above is substantially the same as that of the previous theorem. (2.2) is used in place of (2.1) in the appropriate spot. Similarly we can prove

THEOREM 3.3. Given $f: X \rightarrow Y_{n+1}$. Let $g: X \rightarrow Y_{n+1}$ be any map such that 
$f_{(j)} \cong g_{(j)}$. Then $f_{(j+1)}^{*}=g_{(j+1)}^{*}$ and $\left(f_{(j+1)}\right)_{\#}=\left(g_{(j+1)}\right)_{\#}$ together $\Rightarrow f_{(j+1)} \cong$ $g_{(j+1)}$ for all such $g$, if and only if

$$
\left[\operatorname{ker}\left(k_{j}^{n+1} \mid X\right) \cap \operatorname{ker} \mathscr{H}_{j} \cap \operatorname{ker} \mathscr{G}_{j}\right] / \mathcal{O}^{j}(f, f)=0 .
$$

In the preceding theorems if we set $j=n-1$, then $k_{n-1}^{n+1}$ is a primary cohomology operation. Furthermore, if we now let $n$ vary, we can inductively lift from the lowest stage of a homotopy decomposition up to any other stage, and we have the following:

COROllary 3.4. Assume either $Y$ is a simply connected $H$-space or $Y$ is 2-connected. Then if $f: X \rightarrow Y$ we have

$\left\{\forall n>2, \forall g: X \rightarrow Y_{n}\right.$ such that $g$ can be lifted to $Y_{n+1}$,

$$
\begin{gathered}
\left.g^{*}=\left(f_{(n)}\right)^{*} \Rightarrow g \cong f_{(n)}\right\} \\
\Leftrightarrow\left[\operatorname{ker}\left(k_{j}^{j+2} \mid X\right) \cap \operatorname{ker} \mathscr{H}_{j}\right] / \mathcal{O}^{j}(f, f)=0 \text { for all } j>2 .
\end{gathered}
$$

COROLlaRY 3.5. Assume either $Y$ is a simply connected $H$-space or $Y$ is 2-connected. Then if $f: X \rightarrow Y$ we have

$\left\{\forall n>2, \forall g: X \rightarrow Y_{n}\right.$ such that $g$ can be lifted to $Y_{n+1}$,

$$
\begin{gathered}
\left.g_{\#}=\left(f_{(n)}\right)_{\#} \Rightarrow g \cong f_{(n)}\right\} \\
\Leftrightarrow\left[\operatorname{ker}\left(k_{j}^{j+2} \mid X\right) \cap \operatorname{ker} \mathscr{G}_{j}\right] / \mathcal{O}^{j}(f, f)=0 \text { for all } j \geqq 2 .
\end{gathered}
$$

In the following $f-g$ is composition by suspension or loop-space properties, whichever is appropriate. If $X$ is a suspension then $(f-g)^{*}=$ $f^{*}-g^{*}$ and if $Y$ is a loop-space $(f-g)_{\sharp}=f_{\sharp}-g_{\sharp}$. If $Y$ is a loop-space with homology groups of finite type, then Thomas [8] has shown that $(f-g)^{*}=0 \Leftrightarrow f^{*}=g^{*}$. In all the above cases we have $(f-g) \cong * \Leftrightarrow f \cong g$. For these special cases we derive classification conditions which are not different for each given map $f: X \rightarrow Y$, i.e. do not involve $\mathcal{O}^{j}(f, f)$. These results do involve a knowledge of $\mathcal{O}^{j}(*, *)$ so before stating the obvious corollaries we develop a useful characterization of $\mathcal{O}^{j}(*, *)$. This will also be valuable in the main theorems if $f=^{*}$ and we desire to characterize maps which are null-homotopic.

The obstruction $\mathcal{O}^{n}(f, g)$ to a homotopy from $f$ to $g$ is defined from the following diagram

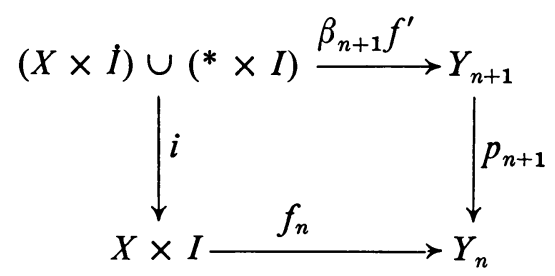

where $f^{\prime}\left|X \times 0=f, f^{\prime}\right| X \times 1=g,\left.f^{\prime}\right|^{*} \times I=*$, and $f_{n}$ is any map making the 
diagram commute. Then

$\mathcal{O}^{n}(f, g)=\Delta^{-1} \lambda_{*}\left\{\left(f_{n}, \beta_{n+1} f^{\prime}\right)^{*} e^{n+1} \mid\right.$ all $f_{n}$ making the diagram commute $\}$ where $e^{n+1} \in H^{n+1}\left(p_{n+1} ; \pi_{n+1}\left(p_{n+1}\right)\right)$ is the fundamental class of $p_{n+1}$, $\lambda: \pi_{n+1}\left(p_{n+1}\right) \approx \pi_{n}(Y)$ induces $\lambda_{*}: H^{n+1}\left(i ; \pi_{n+1}\left(p_{n+1}\right)\right) \rightarrow H^{n+1}\left(i ; \pi_{n}(Y)\right)$, and $\Delta$ is the natural isomorphism $H^{n}\left(X ; \pi_{n}(Y)\right) \rightarrow H^{n+1}\left(i ; \pi_{n}(Y)\right)$. In the case $f=g=^{*}, \beta_{n+1} f^{\prime}={ }^{*}$ and we are looking at all maps $f_{n}: X \times I \rightarrow Y_{n}$ which take $(X \times I) \cup(* \times I)$ to the basepoint, or equivalently maps on the suspension, $S X$. Under the map

$$
H^{n+1}\left(p_{n+1} ; \pi_{n+1}\left(p_{n+1}\right)\right) \rightarrow H^{n+1}\left(Y_{n} ; \pi_{n+1}\left(p_{n+1}\right)\right) \rightarrow H^{n+1}\left(Y_{n} ; \pi_{n}(Y)\right),
$$

$e^{n+1} \rightarrow k^{n+1}$ where $k^{n+1}$ is the appropriate $k$-invariant. The above gives

$$
\mathcal{O}^{n}\left({ }^{*},{ }^{*}\right)=\left\{z \in H^{n}\left(X ; \pi_{n}(Y)\right) \mid \exists h: S X \rightarrow Y_{n} \text { with } \eta(z)=h^{*}\left(k^{n+1}\right)\right\}
$$

where $\eta: H^{n}\left(X ; \pi_{n}(Y)\right) \rightarrow H^{n+1}\left(S X ; \pi_{n}(Y)\right)$. From this a straightforward calculation gives an alternative description: $\mathcal{O}^{n}\left({ }^{*},{ }^{*}\right)=\operatorname{ker}\left(\left(i_{n+1}\right)\right) \mid X$ where $i_{n+1}: K\left(\pi_{n}(Y), n\right) \rightarrow Y_{n+1}$ induces $\left(\left(i_{n+1}\right)\right): H^{n}\left(-; \pi_{n}(Y)\right) \rightarrow\left[-, Y_{n+1}\right]$.

Using the above characterization of $\mathcal{O}^{n}(*, *)$ gives such results as

COROLlary 3.6. Assume either $Y$ is a simply connected loop space with homology groups of finite type, or $Y$ is 2-connected and $X$ is a suspension. Then

$\left\{\forall n>2, \forall f, g: X \rightarrow Y_{n}\right.$ such that $f$ and $g$ are liftable to $Y_{n+1}$,

$$
\begin{gathered}
\left.f^{*}=g^{*} \Rightarrow f \cong g\right\} \\
\Leftrightarrow\left[\operatorname{ker}\left(k_{j}^{j+2} \mid X\right) \cap \operatorname{ker} \mathscr{H}_{j}\right] / \operatorname{ker}\left(\left(i_{j+1}\right)\right)=0 \quad \forall j>2 .
\end{gathered}
$$

4. Applications. Emery Thomas [8] has defined invariants whose vanishing implies that a map is null-homotopic if all of its induced cohomology homomorphisms are zero. The vanishing of these invariants implies that our invariants,

$$
\left[\operatorname{ker}\left(k_{j}^{j+2} \mid X\right) \cap \operatorname{ker} \mathscr{H}_{j}\right] / \mathcal{O}^{j}\left({ }^{*},{ }^{*}\right),
$$

vanish; see [7]. Thomas' result follows from ours, however the two results are not identical. There are trivial examples where $\pi_{n}(Y)$ is not cyclic, however even when $\pi_{n}(Y)$ is cyclic our result is stronger. An example is found letting $X=S^{5}$ and $Y=\left(S^{3} \cup_{f} e^{5}\right) \cup_{2 g} e^{6}$ where $f$ generates $\pi_{4}\left(S^{3}\right)=Z_{2}$ and $g$ generates $\pi_{5}\left(S^{3} \cup_{f} e^{5}\right)=Z$.

Donald Kahn [1] has assigned groups whose vanishing implies that a map is null-homotopic if all of its induced homotopy homomorphisms are zero. The vanishing of these groups implies that our invariants,

$$
\left[\operatorname{ker}\left(k_{j}^{j+2} \mid X\right) \cap \operatorname{ker} \mathscr{G}_{j}\right] / \mathcal{O}^{j}\left({ }^{*},{ }^{*}\right),
$$

vanish; see [7]. Kahn's result follows from ours, however our result is 
stronger. An example is found letting $X=K\left(Z_{2}, 2\right)$ and $Y$ be the two stage Posnikov system $K\left(Z_{2}, 3 ; Z_{2}, 4 ; \mathrm{Sq}^{2}\right)$.

Applying our main theorems to classifying complex vector bundles gives

THEOREM 4.1. Let $\operatorname{dim} X \leqq 2 n$ and suppose that $\forall j, \forall x \in H^{2 j}(X ; Z)$ such that $(j-1) ! x=0$ we have $i_{2 j+1} x \cong *$ where $i_{2 j+1}: K(Z, 2 j) \rightarrow B U_{2 j+1}$. Then equivalence classes of $n$-dimensional complex vector bundles over $X$ can be classified by their associated sets of Chern classes.

The check that $i_{2 j+1} x \cong *$ often involves a knowledge only of properties of the $k$-invariants of $B U$. This includes Theorem 3.2 of Peterson [6] which assumes that the only torsion in $H^{2 j}(X ; Z)$ is relatively prime to $(j-1)$ !. See [7] for an example showing our result is stronger than that of Peterson's.

Our main theorems are stated for maps into specific stages of a homotopy decomposition. Problems involving maps out of specific skeletons of a space can be put into this form via the following:

THEOREM 4.2. There is a 1-1 correspondence $\varphi:\left[X^{n+1}, Y\right]_{n} \rightarrow\left[X, Y_{n+1}\right]$ where $\left[X^{n+1}, Y\right]_{n}$ means equivalence classes of the form $f \sim g$ iff $\left|X^{n} \cong g\right| X^{n}$. The above can be proved by obstruction theory arguments.

Finally we remark that our main theorems can be stated in the more general context of maps which agree on a given subspace $A \subset X$ and which are mapped into a stage of the homotopy decomposition of a fibration $\beta: Y \rightarrow B$, see [7].

\section{REFERENCES}

1. D. W. Kahn, Maps which induce the zero map on homotopy, Pacific J. Math. 15 (1965), 537-540. MR 32 \#1705.

2. K. Mizuno, Semi cubical theory on higher obstruction, J. Inst. Polytech. Osaka City Univ. Ser. A 11 (1960), 15-22. MR 23 \#A655.

3. P. Olum, Obstructions to extensions and homotopies, Ann. of Math. (2). 52 (1950), 29-30. MR 12, 120.

4. - Lecture notes from obstruction theory seminar, Cornell University, Ithaca, N.Y., 1968.

5. - Factorizations and induced homomorphisms, Advances in Math. 3 (1969), 72-100. MR 38 \#6590.

6. F. P. Peterson, Some remarks on Chern classes, Ann. of Math. (2) 69 (1959), 414-420. MR 21 \#1593.

7. M. Sternstein, Ph.D. Dissertation, Cornell University, Ithaca, N.Y., 1971.

8. E. Thomas, Homotopy classification of maps by cohomology homomorphisms, Trans. Amer. Math. Soc. 111 (1964), 138-151. MR 28 \#3426.

Department of Mathematics, Cornell University, Ithaca, New York 14850

Department of Mathematics, Ithaca College, Ithaca, New York 14850 